\title{
Relapse patterns after radiochemotherapy of glioblastoma with FET PET-guided boost irradiation and simulation to optimize radiation target volume
}

Marc D. Piroth ${ }^{1,5,7^{*}}$, Norbert Galldiks ${ }^{4,5,6}$, Michael Pinkawa ${ }^{1,5}$, Richard Holy ${ }^{1,5,7}$, Gabriele Stoffels ${ }^{4,5}$, Johannes Ermert ${ }^{4,5}$, Felix M. Mottaghy ${ }^{2,5}$, N. Jon Shah ${ }^{3,4,5}$, Karl-Josef Langen ${ }^{2,4,5}$ and Michael J. Eble ${ }^{1,5}$

\begin{abstract}
Background: O-(2-18 F-fluoroethyl)-L-tyrosine-(FET)-PET may be helpful to improve the definition of radiation target volumes in glioblastomas compared with MRI. We analyzed the relapse patterns in FET-PET after a FET- and MRI-based integrated-boost intensity-modulated radiotherapy (IMRT) of glioblastomas to perform an optimized target volume definition.

Methods: A relapse pattern analysis was performed in 13 glioblastoma patients treated with radiochemotherapy within a prospective phase-II-study between 2008 and 2009. Radiotherapy was performed as an integrated-boost intensity-modulated radiotherapy (IB-IMRT). The prescribed dose was 72 Gy for the boost target volume, based on baseline FET-PET (FET-1) and 60 Gy for the MRI-based (MRI-1) standard target volume. The single doses were 2.4 and $2.0 \mathrm{~Gy}$, respectively. Location and volume of recurrent tumors in FET-2 and MRI-2 were analyzed related to initial tumor, detected in baseline FET-1. Variable target volumes were created theoretically based on FET-1 to optimally cover recurrent tumor.

Results: The tumor volume overlap in FET and MRI was poor both at baseline (median 12\%; range 0-32) and at time of recurrence (13\%; 0-100). Recurrent tumor volume in FET-2 was localized to $39 \%$ (12-91) in the initial tumor volume (FET-1). Over the time a shrinking (mean $12(5-26) \mathrm{ml}$ ) and shifting (mean $6(1-10 \mathrm{~mm})$ of the resection cavity was seen. A simulated target volume based on active tumor in FET-1 with an additional safety margin of $7 \mathrm{~mm}$ around the FET-1 volume covered recurrent FET tumor volume (FET-2) significantly better than a corresponding target volume based on contrast enhancement in MRI-1 with a same safety margin of $7 \mathrm{~mm}$ (100\% $(54-100)$ versus $85 \%(0-100) ; p<0.01)$. A simulated planning target volume (PTV), based on FET-1 and additional $7 \mathrm{~mm}$ margin plus $5 \mathrm{~mm}$ margin for setup-uncertainties was significantly smaller than the conventional, MR-based PTV applied in this study (median 160 (112-297) $\mathrm{ml}$ versus 231 (117-386) $\mathrm{ml}, p<0.001)$.

(Continued on next page)
\end{abstract}

\footnotetext{
* Correspondence: marc.piroth@helios-kliniken.de

'Department of Radiation Oncology, University Hospital RWTH Aachen, Aachen, Germany

${ }^{5}$ Jülich-Aachen Research Alliance (JARA) - Section JARA-Brain, Research

Center Jülich, Jülich, Germany

Full list of author information is available at the end of the article
} 
(Continued from previous page)

Conclusions: In this small study recurrent tumor volume in FET-PET (FET-2) overlapped only to one third with the boost target volume, based on FET-1. The shrinking and shifting of the resection cavity may have an influence considering the limited overlap of initial and relapse tumor volume. A simulated target volume, based on FET-1 with $7 \mathrm{~mm}$ margin covered $100 \%$ of relapse volume in median and led to a significantly reduced PTV, compared to MRI-based PTVs. This approach may achieve similar therapeutic efficacy but lower side effects offering a broader window to intensify concomitant systemic treatment focusing distant failures.

Keywords: Glioblastoma, Radiochemotherapy, FET-PET, Relapse patterns, Target volume definition

\section{Introduction}

To date, external fractionated radiotherapy is a mainstay in the multimodal treatment strategy of glioblastomas. The diagnostic method of choice for radiation treatment planning is contrast-enhanced MRI owing to its higher anatomical contrast and spatial resolution compared with $\mathrm{CT}$. The differentiation of glioma tissue from surrounding edema, however, may be difficult with MRI and CT particularly when the tumor is not sharply delineated from normal brain tissue, and when the bloodbrain barrier (BBB) remains intact [1]. Tumor cells have been detected beyond the margins of contrast enhancement, in the perifocal edema, and even in the adjacent normal-appearing brain parenchyma [2, 3]. Furthermore, after neurosurgical resection $\mathrm{BBB}$ disturbances and edema can also be treatment-related and cannot be differentiated from residual tumor or tumor recurrence/ progression using conventional MRI [4]. In order to cover all brain areas potentially infiltrated by the tumor, these difficulties lead to rather large target volumes for radiotherapy of glioblastoma [5-9].

In the last decades, amino acid PET using $\mathrm{O}-\left(2-{ }^{18} \mathrm{~F}-\right.$ fluoroethyl)-L-tyrosine (FET) or L-[methyl- $\left.{ }^{11} \mathrm{C}\right]$ methionine (MET) have been shown to be particularly useful to determine the extent of cerebral gliomas more precisely than conventional MRI alone [10-15]. Incorporating such molecular or "biological" imaging information has generated the radiooncological concept of the so called "biological target volume" (BTV) [16]. A number of centers have started to integrate amino acid imaging into CT- and MRI-based radiotherapy planning, particularly when high-precision radiotherapy is planned or in the setting of dose escalation studies or for the re-irradiation of recurrent tumors [17-21].

Some studies have examined the recurrence pattern of glioblastoma in relation to the planning target volume (PTV), either based on treatment planning including FET-PET [22], on MET uptake in the baseline study without using PET for planning [23] or based on the localization of tumor recurrence using FET-PET [24]. The matching observation of all these studies was that the recurrences occurred mainly within the PTV. These studies raised the question whether the "in-field"- recurrences can be reduced by dose escalation to the FET-based BTV, e.g., as a stereotactic dose escalation or by means of a simultaneous integrated boost.

In a recent prospective phase-II trial we performed an integrated-boost intensity-modulated radiotherapy (IBIMRT) with a dose escalation concept giving 72 Gy in 30 fractions to the boost volume based on preirradiation ${ }^{18} \mathrm{~F}$-FET PET imaging [25]. Compared with historical controls and published MRI-based doseescalation studies, however, no improvement of progression-free or overall survival could be observed.

Despite this disappointing result, there remains the notion to optimize the irradiation volume using FET PET and thus to possibly reduce side effects. Therefore, we reviewed the follow-up data of the patients in the above-mentioned study in order to analyze the overlap between residual tumor in the baseline FET-PET (FET-1) post-surgery and the relapse tumor volumes as detected also by FET-PET (FET-2). Based on the results different radiation target volumes were simulated in order to achieve optimal coverage of the tumors with minimal irradiation volume.

To the best of our knowledge, this is the first study comparing the tumor volume in FET PET and MRI at the time of radiation treatment planning to that of FET PET and MRI at the time of tumor recurrence.

\section{Material and methods}

\section{Ethical consideration}

This study was approved by the university ethics committee at the RWTH Aachen faculty of medicine (Ref. No. EK027/07). All participants had given written informed consent for their participation in the study and for publication of the data.

\section{Patients}

This retrospective analysis is based on a previous prospective phase II study [25]. In that study, 22 patients with primary glioblastoma (median age, 55 years; range, $36-73$ years) were treated with radiotherapy and concomitant temozolomide chemotherapy (RCX) followed by adjuvant temozolomide between 01/2008 and 12/2009 [25]. All patients had pre- and postoperative MRI (T1-, T2- and 
FLAIR-weighted images) and postoperative FET-PET for radiation treatment planning. The respective MRI- and FET-PET scans, initial (FET-PET1/MRI1) and also at time of relapse (FET-PET2/MRI2), were performed on the same day. Thereafter, all patients were treated with an IB-IMRT.

Within the follow-up time of 15 months (range, 334 months) 19 patients presented with tumor recurrence on contrast-enhanced MRI. According to the graduation used by Chan et al. [26], a local, local and distant, and distant only recurrence on MRI was seen in 15,3 , and 1 patient(s), respectively. In 13 patients, a repeated FETPET scan was done so that MRI and PET data were available both at the time of the planning of radiotherapy and at the time of recurrence. These 13 patients were included in this relapse pattern analysis. Due to a poor medical condition at the time of recurrence, in the remaining 6 patients FET-PET could not be obtained.

\section{${ }^{18}$ F-FET PET imaging}

The amino acid ${ }^{18} \mathrm{~F}$-FET was produced via nucleophilic ${ }^{18} \mathrm{~F}$-fluorination with a specific radioactivity of $>200 \mathrm{GBq} /$ $\mu \mathrm{mol}$ as described previously. Dynamic PET studies were acquired up to $50 \mathrm{~min}$ after intravenous injection of $200 \mathrm{MBq}$ FET in 3-dimensional mode and reconstructed as described previously [27]. The subsequent evaluation was based on the summarized FET-PET data from 20 to 40 min post injection.

\section{Radiotherapy}

The clinical target volumes (CTV) and planning target volume (PTV) were defined as previously described [25]. In brief, a CTV1 was defined from the postoperative FET-PET using an autocontouring process using a tumor-to-brain ratio (TBR) of FET uptake $\geq 1.6$, which is equivalent to the BTV as mentioned above. This cut-off is based on a biopsy-controlled study in cerebral gliomas where a TBR of 1.6 separated best tumoral from peritumoral tissue [14]. Further, a CTV2 was defined as the contrast-enhanced area from pre- and postoperative MRI including a safety margin of $1.5 \mathrm{~cm}$ and including the preoperative edema, individually adapted to organs at risk and osseous structures. The PTV1 was based on CTV1 with no additional margin. The PTV2 was generated automatically by adding a $0.5 \mathrm{~cm}$ margin around the CTV2. The whole dose was 72 Gy for the PTV1 and 60 Gy for the PTV2 applied with an IB-IMRT (single doses 2.4 and 2 Gy, respectively).

\section{Analysis of tumor volumes at baseline and at the time of recurrence}

In order to analyze the spatial relationship of tumor volumes derived from contrast enhancement in MRI and FET PET at baseline for radiation treatment planning and at the time of recurrence the corresponding data sets were transferred to the Philips Syntegra ${ }^{\mathrm{Tm}}$ image registration tool. After coregistration of MRI and FETPET scans the different volumes were compared volumetrically. The contouring and volume analysis was performed using the Philips Pinnacle ${ }^{3}$ treatment planning software (Version $8.0 \mathrm{~m}$, Philips Medical Systems, Eindhoven, NL).

The volume of the tumor showing contrast enhancement of Gd-DTPA on T1-weighted MRI was determined in baseline MRI for radiation treatment planning (MRI-1) and at the time of relapse (MRI-2). Correspondingly, the tumor volume of FET uptake with a TBR $\geq 1.6$ was evaluated in the baseline FET PET scan (FET-1) and at the time of recurrence (FET-2).

Intersect tumor volumes of Gd-enhancement in MRI and of increased FET-uptake at baseline (MRI-1 $\cap$ FET-1) and corresponding intersect at the time of relapse (MRI-2 $\cap$ FET-2) were determined.

\section{Analysis of the location of tumor recurrence in relation to PTV1 and PTV2}

The primary aim of this study was to analyze the location of the tumor recurrence in FET PET in relation to the tumor area irradiated with a 72 Gy boost (PTV1) which was based on initial FET PET. Furthermore, the recurrence pattern in FET PET in relation to brain area irradiated with a conventional dose of 60 Gy (PTV2) was also considered. This analysis was based on the evaluation of FET-PET data because increased tracer uptake can be considered as a more reliable parameter to determine metabolically active recurrent tumor than contrast enhancement on MRI [28, 29]. For this purpose the tumor volume and fraction of FET positive recurrent tumor within the area irradiated with 60 Gy (PTV2) and within the boost area irradiated with 72 Gy (PTV1) was determined (Table 2).

\section{Analysis of shifting and shrinking of the resection cavity} The shrinking of the resection cavity was analyzed, measuring the volume of the cavity initial and at time of relapse comparatively. Also, the shifting was analyzed measuring the shift of a manually determined representative center point within the cavity.

\section{Simulation of the optimal target volume to cover potential relapse areas}

Based on FET-PET and MRI at baseline (FET-1 and MRT-1) different target volumes were simulated in order to analyze the coverage of the recurrent tumors in FET-2. Therefore, the surface of baseline tumor volumes in FET1 and of contrast enhancement in MRI-1 were surrounded by expanded volumes at a distance of 5,7 and $10 \mathrm{~mm}$ to generate different target volumes. 


\section{Statistical analysis}

The Wilcoxon test was used to compare the tumor volumes and coverage of recurrent tumor tissue by different simulated PTVs based on FET-PET and contrastenhanced MRI. The global significance level for the statistical test procedure conducted was chosen as $\alpha=5 \%$. Statistical analysis was performed using the SPSS Statistics software (Release 20.0, SPSS Inc., Chicago, IL, USA) software.

\section{Results}

\section{Analysis of tumor volumes at baseline and at the time of} recurrence

The tumor volumes for each patient at baseline (FET-1 and MRI-1) and at the time of recurrence (FET-2 and MRI-2) are shown in Table 1. At baseline, the median tumor volume in FET-PET (FET-1) was significantly larger than that of contrast enhancement on MRI-1 (9 (range $1-63) \mathrm{ml}$ vs. $5(0.6-20) \mathrm{ml} ; p=0.01)$ while there was no significant difference between the tumor volumes of FET-PET and MRI at the time of recurrence (FET-2 and MRI-2; 13 (4-67) ml vs. 19 (4-113) $\mathrm{ml} ; p=0.7)$ The intersect between increased FET uptake (TBR > 1.6) and contrast enhancement in MRI was generally poor both at baseline and at the time of relapse $(12 \%(0-32)$ and $13 \%(0-100)$, respectively). The discrepancy between
FET uptake and contrast enhancement on MRI is illustrated in Fig. $1 \mathrm{~b}$ and $\mathrm{d}$.

\section{Analysis of the location of tumor recurrence in relation to PTV1 and PTV2}

Data on the location of pathological tracer uptake in FET PET in relation to PTV1 and PTV2 at the time of tumor recurrence are shown in Table 2. The fraction of the recurrent FET tumor volume within the 72 Gy boost volume PTV1, was only 39 \% (12-91), i.e., nearly two thirds of recurrent tumor tissue was located outside the boost volume. In contrast, recurrent FET tumor volume was located to $100 \%$ within the large PTV-2 based on conventional MRI which was irradiated by the standard dose of $60 \mathrm{~Gy}$.

Analysis of shifting and shrinking of the resection cavity The resection cavity shrinked by $12 \mathrm{ml}(4.8-26)$ and shifted by $6 \mathrm{~mm}(1-10.3)$ in mean over time.

\section{Simulation of the optimal PTV to cover potential recurrence areas}

The target volumes simulated on the basis of FET-PET after resection (FET-1) exhibited generally better coverage of the recurrent FET tumor volume (FET-2) than the corresponding target volumes simulated on the basis of the

Table 1 Tumor volumes of increased FET-uptake and of Gd-enhancement in MRI at baseline and at time of relapse

\begin{tabular}{|c|c|c|c|c|c|c|c|c|}
\hline \multirow[t]{2}{*}{ Pat. No } & \multicolumn{4}{|c|}{ Tumor volumes at baseline } & \multicolumn{4}{|c|}{ Tumor volumes at relapse } \\
\hline & FET-1 (ml) & MRI-1 (ml) & $\begin{array}{l}\text { Intersect FET-1 } \cap \\
\text { MRI-1 (ml) }\end{array}$ & $\begin{array}{l}\text { Intersect FET- } 1 \cap \\
\text { MRI-1 ( } \% \text { of FET- } 1 \text { ) }\end{array}$ & FET-2 (ml) & MRI-2 (ml) & $\begin{array}{l}\text { Intersect FET-2 } n \\
\text { MRI- } 2(\mathrm{ml})\end{array}$ & $\begin{array}{l}\text { Intersect FET- } 2 \cap \\
\text { MRI-2 (\% of FET-2) }\end{array}$ \\
\hline 1 & 10.4 & 0.8 & 0.4 & 3.8 & 3.7 & 3.7 & 0.0 & 0 \\
\hline 2 & 6.9 & 0.6 & 0.2 & 2.9 & 10.9 & 8.9 & 0.7 & 8.7 \\
\hline 3 & 6.8 & 3.7 & 2.2 & 32.4 & 11.3 & 8.1 & 5.0 & 44.2 \\
\hline 4 & 1.1 & 6.8 & 1.3 & 9.9 & 12.2 & 9.3 & 7.6 & 62.3 \\
\hline 5 & 13.4 & 6.0 & 3.5 & 26.1 & 13.9 & 12.0 & 1.1 & 7.9 \\
\hline 6 & 15.0 & 1.3 & 0.5 & 3.3 & 35.1 & 16.2 & 23.5 & 67.0 \\
\hline 7 & 6.7 & 6.4 & 0.0 & 0.0 & 6.2 & 19.4 & 0.8 & 12.9 \\
\hline 8 & 25.2 & 1.3 & 1.3 & 5.2 & 22.1 & 26.8 & 10.0 & 45.3 \\
\hline 9 & 4.7 & 6.5 & 1.5 & 31.9 & 5.6 & 27.0 & 5.6 & 100 \\
\hline 10 & 6.8 & 4.9 & 1.6 & 23.5 & 56.0 & 39.6 & 0.0 & 0.0 \\
\hline 11 & 62.9 & 19.4 & 12.0 & 19.1 & 46.7 & 41.8 & 23.4 & 50.1 \\
\hline 12 & 59.0 & 19.5 & 11.3 & 19.2 & 66.8 & 43.5 & 5.5 & 8.2 \\
\hline 13 & 9.1 & 1.1 & 1.1 & 12.1 & 12.8 & 113.0 & 1.7 & 13.3 \\
\hline Mean & 17.5 & 6.0 & 3.0 & 14.6 & 23.3 & 28.4 & 6.5 & 32.3 \\
\hline Median & 9.1 & 4.9 & 1.3 & 12.1 & 12.8 & 19.4 & 5.0 & 13.3 \\
\hline SD & 19.6 & 6.1 & 4.0 & 11.5 & 20.9 & 28.8 & 8.1 & 31.5 \\
\hline range & $1.1-62.9$ & $0.6-19.5$ & $0-12$ & $0-32.4$ & $3.7-66.8$ & $3.7-113$ & $0-23.5$ & $0-100.0$ \\
\hline
\end{tabular}

FET-1: pathological FET-Volume in $\mathrm{ml}$ at baseline (post surgery) MRI-1: Gd-contrast-enhancement in $\mathrm{ml}$ at baseline (post surgery) FET-2: pathological FET-Volume in $\mathrm{ml}$ at the time of relapse MRI-1: Gd-contrast-enhancement in $\mathrm{ml}$ at the time of relapse 


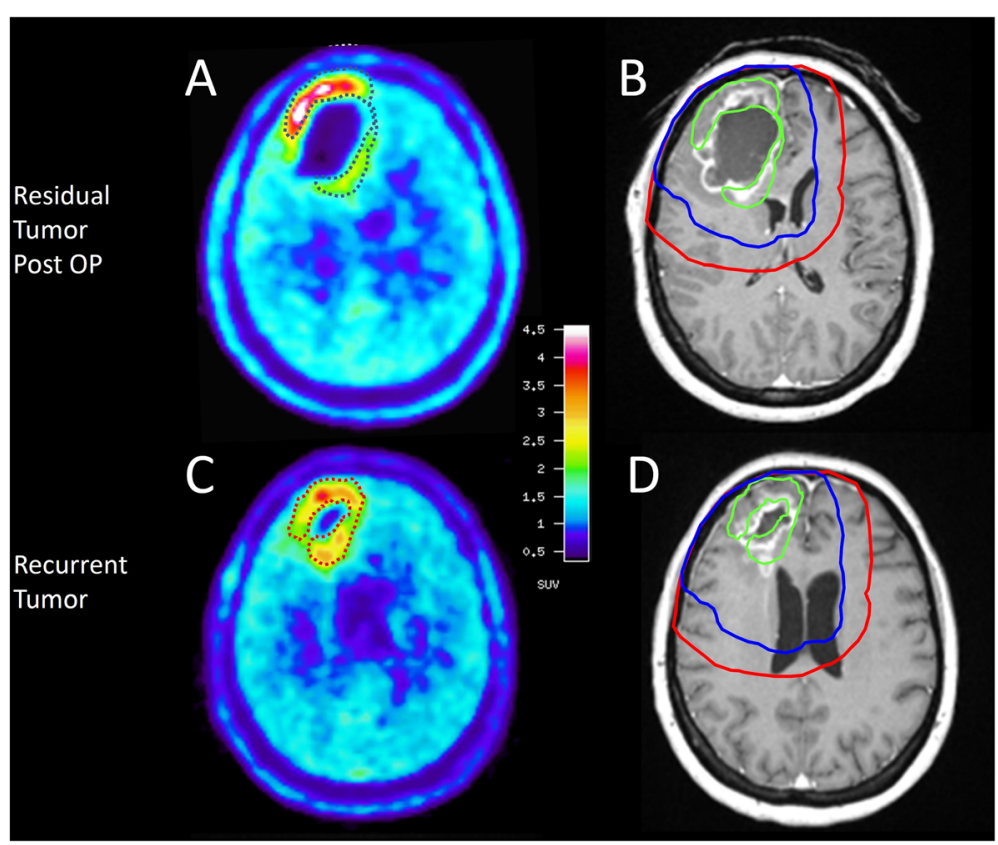

Fig. 1 Residual tumor volumes in FET-PET and MRI after glioblastoma resection left frontal are shown in the upper row $(\mathbf{a}, \mathbf{b})$ and of the recurrent tumor in the lower row $(\mathbf{c}, \mathbf{d})$. The tumor volume with increased FET uptake is surrounded by a dotted line in FET-PET (a, $\mathbf{c})$ and by a green line in contrast-enhanced MRI (b, d). Note the discrepancy between FET uptake and contrast enhancement both in the baseline scan (b) and at the time of relapse (d). The definition of PTV2, which is based on MRI, is indicated by the red line (b, d). The blue line demonstrated a simulated PTV based on a CTV consisting of FET-1 plus $7 \mathrm{~mm}$ margin

Table 2 FET-uptake at time of relapse in relation to PTV-1 and PTV-2

\begin{tabular}{lllll}
\hline Pat. No & FET Tumor volume at relapse & PTV-1 (72 Gy) & PTV-2 (60 Gy) \\
\cline { 3 - 5 } & FET-2 $(\mathrm{ml})$ & Part of FET-2 in PTV-1 (ml) & Fraction of FET-2 in PTV-1 (\%) & Fraction of FET-2 in PTV-2 (\%) \\
\hline 1 & 3.7 & 1.2 & 11.5 & 100 \\
2 & 10.9 & 1.1 & 15.9 & 100 \\
3 & 11.3 & 3.8 & 55.9 & 100 \\
4 & 12.2 & 5.1 & 38.9 & 100 \\
5 & 13.9 & 4.9 & 36.6 & 100 \\
6 & 35.1 & 13.7 & 91.3 & 100 \\
7 & 6.2 & 1.6 & 23.9 & 100 \\
8 & 22.1 & 9.5 & 37.7 & 100 \\
9 & 5.6 & 0.6 & 12.8 & 100 \\
10 & 56.0 & 2.8 & 41.2 & 100 \\
11 & 46.7 & 29.4 & 46.7 & 100 \\
12 & 66.8 & 29.0 & 49.2 & 100 \\
13 & 12.8 & 4.4 & 48.4 & 100 \\
Mean & 23.3 & 8.2 & 39.2 & 100 \\
Median & 12.8 & 4.4 & 38.9 & 21.4 \\
SD & 20.9 & 10.0 & $11.5-91.3$ &
\end{tabular}


contrast-enhanced MRI (Table 3). Thus theoretically, a CTV based on FET-1 without any margin showed a significant better coverage of FET-2 than a corresponding target volume based on contrast enhancement in MRI-1 (median $34 \%(5-63)$ vs. $21 \%(0-42) ; p<0.01)$, FET- 1 and MRI-1 with a margin of $5 \mathrm{~mm}(94 \%(42-100)$ vs. $74 \%$ $(0-92) ; p<0.01)$, FET-1 and MRI-1 with a margin of $7 \mathrm{~mm}(100 \%(54-100)$ vs. $85 \%(0-100) ; p<0.01)$, FET- 1 and MRI-1 with a margin of $10 \mathrm{~mm}(100 \%(82-100)$ vs. $86 \%(0-100) ; p<0.01)$.

The resulting simulated PTVs on the basis of FETPET after surgery with different margins in comparison with the actual PTV-2 from the study are shown in Table 4.

An optimal compromise appears to be a CTV based on FET-1 with a margin of $7 \mathrm{~mm}$ because there is a high coverage of recurrent tumor volume in FET-PET (100\% (54-100)) and a significantly smaller PTV compared to a typical MRI-based PTV performed in our study (160 $(112-297) \mathrm{ml}$ vs. $231(117-386) \mathrm{ml}, p<0.001)$.

\section{Discussion}

To date, the definition of the optimal target volume in radiation treatment planning of glioblastomas is controversial [30,31]. According to current standards, target volume concepts are based on either preoperative or postoperative MRIs, which, however, lead to relative large target volumes [5-9]. PET using radiolabeled amino acids such as FET can offer a more precise delineation of the metabolically active tumor, which is not limited to the area of $\mathrm{BBB}$ disruption and is more specific than the information provided by conventional MRI alone [14, 32, 33]. A number of centers have started to integrate the BTV as depicted by amino acid PET into CTand MRI-based radiotherapy planning [12, 17-20, 24]. Considerable discrepancies between the PTVs arising from MRI and PET have been demonstrated in several studies $[12,17,19,24,34]$.

In addition to the observed differences in the extent of the tumor in MRI and PET in radiotherapy planning, the localization and the definition of the extent of the recurrent tumor is another diagnostic problem. Treatment-related BBB alterations with consecutive contrast enhancement on conventional MRI can mimic tumor recurrence and are difficult to differentiate from progressive tumor. It has been shown in several studies that FET PET is more reliable to differentiate tumor tissue in recurrent gliomas and posttherapeutic changes in the tissue than conventional MRI [11, 28, 35].

Some studies have examined the recurrence pattern of glioblastoma taking into account amino acid PET in various ways. One study included FET-PET for RT planning but the location of recurrences was evaluated by contrast enhanced MRI only [22]. Another study analyzed

Table 3 Coverage of recurrent FET tumor volume by different simulated CTVs based on PET/MRI at baseline

\begin{tabular}{|c|c|c|c|c|c|c|c|c|}
\hline \multirow[t]{2}{*}{ CTV } & MRI-1 & FET-1 & MRI-1 & FET-1 & MRI-1 & FET-1 & MRI-1 & FET-1 \\
\hline & \multicolumn{2}{|c|}{ No margin } & \multicolumn{2}{|c|}{$+5 \mathrm{~mm}$ margin } & \multicolumn{2}{|c|}{$+7 \mathrm{~mm}$ margin } & \multicolumn{2}{|c|}{$+10 \mathrm{~mm}$ margin } \\
\hline 1 & 0,27 & 0,33 & 0,8 & 0,95 & 0,87 & 1,00 & 0,93 & 1,00 \\
\hline 2 & 0,08 & 0,098 & 0,49 & 0,61 & 0,52 & 0,76 & 0,7 & 1,00 \\
\hline 3 & 0,21 & 0,34 & 0,61 & 0,95 & 0,67 & 1,00 & 0,83 & 1,00 \\
\hline 4 & 0,33 & 0,42 & 0,74 & 0,94 & 0,85 & 1,00 & 0,86 & 1,00 \\
\hline 5 & 0,25 & 0,35 & 0,92 & 0,96 & 0,99 & 1,00 & 1,00 & 1,00 \\
\hline 6 & 0,05 & 0,39 & 0,19 & 0,85 & 0,23 & 0,92 & 0,33 & 0,972 \\
\hline 7 & 0 & 0,25 & 0 & 0,831 & 0 & 0,91 & 0 & 0,98 \\
\hline 8 & 0,2 & 0,32 & 0,76 & 0,93 & 1,00 & 1,00 & 1,00 & 1,00 \\
\hline 9 & 0,08 & 0,11 & 0,79 & 0,84 & 0,88 & 0,91 & 0,9 & 0,95 \\
\hline 10 & 0,16 & 0,05 & 0,55 & 0,42 & 0,62 & 0,54 & 0,84 & 0,82 \\
\hline 11 & 0,42 & 0,63 & 0,51 & 0,96 & 0,578 & 0,97 & 0,77 & 1,00 \\
\hline 12 & 0,3 & 0,44 & 0,79 & 1,00 & 0,90 & 1,00 & 0,96 & 1,00 \\
\hline 13 & 0,22 & 0,34 & 0,91 & 1,00 & 1,00 & 1,00 & 1,00 & 1,00 \\
\hline mean & 0,20 & 0,31 & 0,62 & 0,86 & 0,70 & 0,92 & 0,78 & 0,98 \\
\hline median & 0,21 & 0,34 & 0,74 & 0,94 & 0,85 & 1 & 0,86 & 1 \\
\hline SD & 0,12 & 0,16 & 0,27 & 0,17 & 0,31 & 0,13 & 0,39 & 0,05 \\
\hline range & $0-0,42$ & $0,05-0,63$ & $0-0,92$ & $0,42-1$ & $0-1$ & $0,54-1$ & $0-1$ & $0,82-1$ \\
\hline
\end{tabular}

CTV Clinical Target Volume

FET-1 pathological FET-Volume in $\mathrm{ml}$ at baseline (post surgery) MRI-1: Gd-contrast-enhancement in $\mathrm{ml}$ at baseline (post surgery) $S D$ Standard Deviation 
Table 4 Volumes of standard and simulated PTVs on the basis of FET-PET

\begin{tabular}{|c|c|c|c|c|}
\hline Pat. No. & $\begin{array}{l}\text { PTV-2 MRI } \\
\text { standard (ml) }\end{array}$ & $\begin{array}{l}\text { PTV FET-1 } \\
+5 \mathrm{~mm} \text { margin (ml) }\end{array}$ & $\begin{array}{l}\text { PTV FET-1 } \\
+7 \text { mm margin (ml) }\end{array}$ & $\begin{array}{l}\text { PTV FET-1 } \\
+10 \mathrm{~mm} \text { margin }(\mathrm{ml})\end{array}$ \\
\hline 1 & 245 & 122 & 147 & 214 \\
\hline 2 & 164 & 98 & 118 & 163 \\
\hline 3 & 117 & 92 & 112 & 143 \\
\hline 4 & 248 & 125 & 150 & 194 \\
\hline 5 & 161 & 101 & 114 & 146 \\
\hline 6 & 256 & 182 & 213 & 286 \\
\hline 7 & 224 & 106 & 126 & 166 \\
\hline 8 & 348 & 216 & 253 & 308 \\
\hline 9 & 209 & 161 & 187 & 259 \\
\hline 10 & 160 & 138 & 160 & 216 \\
\hline 11 & 364 & 233 & 263 & 304 \\
\hline 12 & 386 & 265 & 297 & 342 \\
\hline 13 & 231 & 175 & 197 & 258 \\
\hline mean & 240 & 155 & 180 & 230 \\
\hline median & 231 & 138 & 160 & 216 \\
\hline SD & 83 & 56 & 62 & 67 \\
\hline Range & $117-386$ & $92-265$ & $112-297$ & $143-342$ \\
\hline
\end{tabular}

(Volumes of conventional PTV2 and simulated PTVs based on FET uptake at baseline (FET-1) expanded by 5, 7 and 10 mm margin in ml. The PTV include an additional margin of $5 \mathrm{~mm}$ around the CTV which is standardly used to compensate the set-up-and immobilisation uncertainties)

PTV Planning Target Volume

FET-1: pathological FET-Volume in $\mathrm{ml}$ at baseline (post surgery)

MRI-1:Gd-contrast-enhancement in $\mathrm{ml}$ at baseline (post surgery)

SD Standard Deviation

the location of recurrences in contrast-enhanced MRI in comparison to MET uptake in the baseline study without using PET for treatment planning [23]. A recent study investigated the localization of tumor recurrence in FETPET after re-irradiation with bevacizumab in recurrent malignant gliomas [24]. The matching observation of all these studies was that the recurrences occurred mainly within the PTV but it has to be considered that in no study amino acid PET was available in both the baseline study and at the time of relapse.

In this retrospective study we analyzed relapse patterns of glioblastoma in FET-PET and MRI after IB-IMRT with dose escalation based on FET-PET.

A first aspect in this study was the comparison of the extent of contrast enhancement on MRI to that of FET uptake in the baseline study and at the time of recurrence. In agreement with previous studies the intersection between pathological FET uptake and contrast enhancement in MRI was generally poor both at baseline and at the time of recurrence. This observation confirms the view that contrast enhancement in MRI does not reliably reflect the extent of the metabolically active tumor volume and should be therefore considered with caution $[12,17,19$, 24, 34]. Tumor volumes in FET-PET and contrastenhanced MRI were not significantly different at the time of relapse and the overlap was $13 \%$ in median only.
The comparison of the relapse volume in FET-PET in relation to PTV2 demonstrated that $100 \%$ of the tumor recurrences were located in the routinely performed large target volumes using MRI based treatment planning [5-9]. This is in agreement with the results of previous studies including PET data [22-24] and is also in accordance with the literature based on conventional imaging where all local relapses were detected within the volume enclosed by the $95 \%$ isodosis line of the prescribed dose of 60 Gy [26, 36, 37]. This is not unexpected, since radiation treatment planning based on MRI scans usually encompass the resection cavity and the contrast enhancing area with a margin up to $3 \mathrm{~cm}$ [5], resulting in large radiation target volumes.

Comparison of the relapse volume in FET-PET in relation to the boost target volume applied in our study, however, revealed that more than two thirds of recurrent tumor tissue in FET-PET was located outside the boost volume. The limited overlap may be influenced by the shifts of brain tissue due to shrinkage of the resection cavity seen in our analysis (see Fig. 1) but the difference is considerable and cannot be explained solely by these factors. Therefore it can be assumed that a large proportion of recurrences have grown outside the boost volumes i.e. within the area of the prescribed dose of 60 Gy. 
Based on this assumption we simulated different CTVs on the basis of FET-PET in order to analyze the coverage of the recurrent tumors in FET-PET. The CTVs simulated on the basis of FET-PET after surgery exhibited generally better coverage of the recurrent FET tumor volume than the corresponding CTVs simulated on the basis of the contrast-enhanced MRI. Using a CTV based on FET-1 with a margin of $7 \mathrm{~mm}$ achieved a high coverage of recurrent tumor volume in FET-PET of $100 \%$ (54-100). Accordingly, a significantly smaller PTV results compared to the conventional MR-based PTV used in this study (160 (112-297) $\mathrm{ml}$ vs. $231(117-386) \mathrm{ml}$, $p<0.001)$. This analysis indicates that a PTV based on FET-PET may achieve a coverage which is at least comparable to standard MRI-based PTVs but less toxic considering the shown PTV reduction. This approach may help to achieve similar therapeutic efficacy but lower side effects. This may be of interest with regard to an intensification of concomitant systemic treatment schemes probably required to improve outcome. Furthermore, sparing of larger parts of the brain increases the systemic treatment options in the case of distant recurrences.

\section{Conclusion}

Overlap of pathological FET uptake in glioblastoma and contrast enhancement in MRI was generally poor both at baseline and at the time of relapse. Relapse volumes of the tumor recurrences in FET-PET were located to $100 \%$ in PTV2 achieving 60 Gy, but more nearly two thirds was located outside the boost volume PTV1. A CTV based on FET with a safety margin of $7 \mathrm{~mm}$ covers $100 \%$ of the relapse volume and consecutively reduces the PTV significantly. This approach may achieve similar therapeutic efficacy but lower side effects and offer options to intensify concomitant systemic treatment focusing the problem of distant failures. Because of the small sample size further studies are needed to confirm these findings.

\section{Ethical consideration and consent to participate}

The study was approved by the university ethics committee at the RWTH Aachen faculty of medicine (Ref. No. EK027/07). All participants had given written informed consent for their participation in the study.

\section{Consent for publication}

Not applicable.

\section{Availability of data and materials}

The datasets supporting the conclusions of this article are included within the article.

\section{Abbreviations}

BBB, blood-brain barrier; BTV, biological target volume; $C T$, computer tomography; CTV, clinical target volume; FET, O-(2-18 F-fluoroethyl)-L-tyrosine; FLAIR, fluid-attenuated inversion recovery; Gd-DTPA, Gadolinium-

diethylenetriaminepentacetate; IB-IMRT, integrated boost-intensity-modulated radiotherapy; IMRT, intensity-modulated radiotherapy; MET, L-(methyl-11C)methionine; $\mathrm{MRl}$, magnetic resonance imaging; $\mathrm{PET}$, positron emission tomography; PTV, planning target volume; RCX, radio-chemotherapy; TBR, tumor-to-brain ratio.

\section{Competing interests}

The authors declare that they have no competing interests.

\section{Authors' contributions}

MDP has made substantial contributions to the conception, acquisition of data, analysis and interpretation of data and drafted the manuscript. NG has made substantial contributions to the conception, acquisition of data, analysis and interpretation of data and helped to draft the manuscript. MP has been involved in acquisition of data and helped to draft the manuscript. $\mathrm{RH}$ has been involved in acquisition of data and helped to draft the manuscript. GS has been involved in acquisition of data and helped to draft the manuscript. JE was responsible for tracer production and quality control and has been involved in acquisition of data. FMM made contributions to the conception and helped to draft the manuscript. NJS made contributions to the conception and acquisition of data and helped to draft the manuscript. KLL has made substantial contributions to the conception, acquisition of data, analysis and interpretation of data and helped to draft the manuscript. MJE has made substantial contributions to the conception, acquisition of data, analysis and interpretation of data and helped to draft the manuscript. All authors read and approved the final manuscript.

\section{Acknowledgements}

We would like to thank the staff who took care of our patients' needs, and who were involved in gathering, documenting, verifying, forwarding and processing the clinical data.

\section{Funding}

None.

\section{Author details}

'Department of Radiation Oncology, University Hospital RWTH Aachen, Aachen, Germany. ${ }^{2}$ Department of Nuclear Medicine, University Hospital RWTH Aachen, Aachen, Germany. ${ }^{3}$ Department of Neurology, University Hospital RWTH Aachen, Aachen, Germany. ${ }^{4}$ Institute of Neuroscience and Medicine, Research Center Jülich, Jülich, Germany. ${ }^{5}$ Jülich-Aachen Research Alliance (JARA) - Section JARA-Brain, Research Center Jülich, Jülich, Germany.

${ }^{6}$ Department of Neurology, University of Cologne, Cologne, Germany. ${ }^{7}$ Department of Radiation Oncology, Faculty of Health, Witten/Herdecke University, HELIOS Hospital Wuppertal Heusnerstr, 4042283 Wuppertal, Germany.

Received: 1 January 2016 Accepted: 23 June 2016

Published online: 24 June 2016

\section{References}

1. Jansen EP, Dewit LG, van Herk M, Bartelink H. Target volumes in radiotherapy for high-grade malignant glioma of the brain. Radiother Oncol. 2000;56(2):151-6.

2. Halperin EC, Bentel G, Heinz ER, Burger PC. Radiation therapy treatment planning in supratentorial glioblastoma multiforme: an analysis based on post mortem topographic anatomy with $C T$ correlations. Int J Radiat Oncol Biol Phys. 1989;17(6):1347-50.

3. Lunsford LD, Martinez AJ, Latchaw RE. Magnetic resonance imaging does not define tumor boundaries. Acta Radiol Suppl. 1986;369:154-6.

4. Wen PY, Macdonald DR, Reardon DA, Cloughesy TF, Sorensen AG, Galanis E, Degroot J, Wick W, Gilbert MR, Lassman AB, et al. Updated response assessment criteria for high-grade gliomas: response assessment in neuro-oncology working group. JClinOncol. 2010;28(11):1963-72.

5. Chang EL, Akyurek S, Avalos T, Rebueno N, Spicer C, Garcia J, Famiglietti R, Allen PK, Chao KS, Mahajan A, et al. Evaluation of peritumoral edema in the delineation of radiotherapy clinical target volumes for glioblastoma. Int J Radiat Oncol Biol Phys. 2007;68(1):144-50. 
6. McDonald MW, Shu HK, Curran Jr WJ, Crocker IR. Pattern of failure after limited margin radiotherapy and temozolomide for glioblastoma. Int J Radiat Oncol Biol Phys. 2011;79(1):130-6.

7. Stupp R, Mason WP, van den Bent MJ, Weller M, Fisher B, Taphoorn MJ, Belanger K, Brandes AA, Marosi C, Bogdahn U, et al. Radiotherapy plus concomitant and adjuvant temozolomide for glioblastoma. N Engl J Med. 2005;352(10):987-96.

8. Aydin $\mathrm{H}$, Sillenberg I, von Lieven $\mathrm{H}$. Patterns of failure following CT-based 3-D irradiation for malignant glioma. Strahlenther Onkol. 2001;177(8):424-31.

9. Mason WP, Maestro RD, Eisenstat D, Forsyth P, Fulton D, Laperriere N, Macdonald D, Perry J, Thiessen B, Canadian GBMRC. Canadian recommendations for the treatment of glioblastoma multiforme. Curr Oncol. 2007;14(3):110-7.

10. Galldiks N, Ullrich R, Schroeter M, Fink GR, Jacobs AH, Kracht LW. Volumetry of [(11)C]-methionine PET uptake and MRI contrast enhancement in patients with recurrent glioblastoma multiforme. Eur J Nucl Med Mol Imaging. 2010;37(1):84-92.

11. Galldiks N, Langen KJ, Holy R, Pinkawa M, Stoffels G, Nolte KW, Kaiser HJ, Filss CP, Fink GR, Coenen HH, et al. Assessment of treatment response in patients with glioblastoma using O-(2-18 F-fluoroethyl)-L-tyrosine PET in comparison to MRI. J Nucl Med. 2012;53(7):1048-57.

12. Grosu AL, Weber WA, Riedel E, Jeremic B, Nieder C, Franz M, Gumprecht H, Jaeger R, Schwaiger M, Molls M. L-(methyl-11C) methionine positron emission tomography for target delineation in resected high-grade gliomas before radiotherapy. Int J Radiat Oncol Biol Phys. 2005;63(1):64-74.

13. Miwa K, Shinoda J, Yano H, Okumura A, Iwama T, Nakashima T, Sakai N. Discrepancy between lesion distributions on methionine PET and MR images in patients with glioblastoma multiforme: insight from a PET and MR fusion image study. J Neurol Neurosurg Psychiatry. 2004;75(10):1457-62.

14. Pauleit D, Floeth F, Hamacher K, Riemenschneider MJ, Reifenberger G, Muller HW, Zilles K, Coenen HH, Langen KJ. O-(2-[18 F]fluoroethyl)-Ltyrosine PET combined with MRI improves the diagnostic assessment of cerebral gliomas. Brain. 2005;128(Pt 3):678-87.

15. Piroth MD, Holy R, Pinkawa M, Stoffels G, Kaiser HJ, Galldiks N, Herzog H, Coenen HH, Eble MJ, Langen KJ. Prognostic impact of postoperative, pre-irradiation 18F-Fluoroethyl-L-Tyrosine uptake in glioblastoma patients treated with radiochemotherapy. Radiother Oncol. 2011;99(2):2018-24.

16. Ling CC, Humm J, Larson S, Amols H, Fuks Z, Leibel S, Koutcher JA. Towards multidimensional radiotherapy (MD-CRT): biological imaging and biological conformality. Int J Radiat Oncol Biol Phys. 2000;47(3):551-60.

17. Levivier M, Massager N, Wikler D, Goldman S. Modern multimodal neuroimaging for radiosurgery: the example of PET scan integration. Acta Neurochir Suppl. 2004;91:1-7.

18. Rickhey M, Koelbl O, Eilles C, Bogner L. A biologically adapted doseescalation approach, demonstrated for 18 F-FET-PET in brain tumors. Strahlenther Onkol. 2008;184(10):536-42.

19. Weber DC, Zilli T, Buchegger F, Casanova N, Haller G, Rouzaud M, Nouet P, Dipasquale G, Ratib O, Zaidi H, et al. [(18)F]Fluoroethyltyrosine- positron emission tomography-guided radiotherapy for high-grade glioma. Radiat Oncol. 2008;3:44

20. Piroth MD, Pinkawa M, Holy R, Stoffels G, Demirel C, Attieh C, Kaiser HJ, Langen KJ, Eble MJ. Integrated-boost IMRT or 3-D-CRT using FET-PET based auto-contoured target volume delineation for glioblastoma multiforme-a dosimetric comparison. Radiat Oncol. 2009;4:57.

21. Grosu AL, Weber WA, Franz M, Stark S, Piert M, Thamm R, Gumprecht H, Schwaiger M, Molls M, Nieder C. Reirradiation of recurrent high-grade gliomas using amino acid PET (SPECT)/CT/MRI image fusion to determine gross tumor volume for stereotactic fractionated radiotherapy. Int J Radiat Oncol Biol Phys. 2005;63(2):511-9.

22. Weber DC, Casanova N, Zilli T, Buchegger F, Rouzaud M, Nouet P, Vees $H_{\text {, }}$ Ratib O, Dipasquale G, Miralbell R. Recurrence pattern after [(18)F]fluoroethyltyrosine-positron emission tomography-guided radiotherapy for high-grade glioma: a prospective study. Radiother Oncol. 2009;93(3):586-92.

23. Lee $I_{\text {, }}$ Piert M, Gomez-Hassan D, Junck L, Rogers L, Hayman J, Ten Haken RK, Lawrence TS, Cao Y, Tsien C. Association of 11C-methionine PET uptake with site of failure after concurrent temozolomide and radiation for primary glioblastoma multiforme. Int J Radiat Oncol Biol Phys. 2009;73(2):479-85.

24. Niyazi M, Jansen NL, Rottler M, Ganswindt U, Belka C. Recurrence pattern analysis after re-irradiation with bevacizumab in recurrent malignant glioma patients. Radiat Oncol. 2014;9:299.
25. Piroth MD, Pinkawa M, Holy R, Klotz J, Schaar S, Stoffels G, Galldiks N, Coenen HH, Kaiser HJ, Langen KJ, et al. Integrated boost IMRT with FET-PETadapted local dose escalation in glioblastomas. Results of a prospective phase II study. Strahlenther Onkol. 2012;188(4):334-9.

26. Chan JL, Lee SW, Fraass BA, Normolle DP, Greenberg HS, Junck LR, Gebarski SS, Sandler HM. Survival and failure patterns of high-grade gliomas after three-dimensional conformal radiotherapy. J Clin Oncol. 2002;20(6):1635-42.

27. Langen KJ, Hamacher K, Weckesser M, Floeth F, Stoffels G, Bauer D, Coenen HH, Pauleit D. O-(2-[18 F]fluoroethyl)-L-tyrosine: uptake mechanisms and clinical applications. Nucl Med Biol. 2006;33(3):287-94.

28. Galldiks N, DunkI V, Stoffels G, Hutterer M, Rapp M, Sabel M, Reifenberger G, Kebir S, Dorn F, Blau T, et al. Diagnosis of pseudoprogression in patients with glioblastoma using O-(2-[18 F]fluoroethyl)-L-tyrosine PET. Eur J Nucl Med Mol Imaging. 2015:42(5):685-95.

29. Galldiks N, Stoffels G, Filss C, Rapp M, Blau T, Tscherpel C, Ceccon G, Dunkl $V$, Weinzierl M, Stoffel M, et al. The use of dynamic O-(2-18 F-fluoroethyl)-Ityrosine PET in the diagnosis of patients with progressive and recurrent glioma. Neuro-Oncology. 2015;17(9):1293-300.

30. Farace P, Giri MG, Meliado G, Amelio D, Widesott L, Ricciardi GK, Dall'Oglio S, Rizzotti A, Sbarbati A, Beltramello A, et al. Clinical target volume delineation in glioblastomas: pre-operative versus post-operative/preradiotherapy MRI. Br J Radiol. 2011:84(999):271-8.

31. Minniti G, Amelio D, Amichetti M, Salvati M, Muni R, Bozzao A, Lanzetta G, Scarpino S, Arcella A, Enrici RM. Patterns of failure and comparison of different target volume delineations in patients with glioblastoma treated with conformal radiotherapy plus concomitant and adjuvant temozolomide. Radiother Oncol. 2010;97(3):377-81

32. Kracht LW, Miletic H, Busch S, Jacobs AH, Voges J, Hoevels M, Klein JC, Herholz K, Heiss WD. Delineation of brain tumor extent with [11C]Lmethionine positron emission tomography: local comparison with stereotactic histopathology. Clin Cancer Res. 2004;10(21):7163-70.

33. Pafundi DH, Laack NN, Youland RS, Parney IF, Lowe VJ, Giannini C, Kemp BJ, Grams MP, Morris JM, Hoover JM, et al. Biopsy validation of 18 F-DOPA PET and biodistribution in gliomas for neurosurgical planning and radiotherapy target delineation: results of a prospective pilot study. Neuro-Oncology. 2013;15(8):1058-67.

34. Rieken S, Habermehl D, Giesel FL, Hoffmann C, Burger U, Rief H, Welzel T, Haberkorn U, Debus J, Combs SE. Analysis of FET-PET imaging for target volume definition in patients with gliomas treated with conformal radiotherapy. Radiother Oncol. 2013;109(3):487-92.

35. Popperl G, Gotz C, Rachinger W, Gildehaus FJ, Tonn JC, Tatsch K. Value of O-(2-[18 F]fluoroethyl)- L-tyrosine PET for the diagnosis of recurrent glioma. Eur J Nucl Med Mol Imaging. 2004;31(11):1464-70.

36. Lee SW, Fraass BA, Marsh LH, Herbort K, Gebarski SS, Martel MK, Radany EH, Lichter AS, Sandler HM. Patterns of failure following high-dose 3-D conformal radiotherapy for high-grade astrocytomas: a quantitative dosimetric study. Int J Radiat Oncol Biol Phys. 1999;43(1):79-88.

37. Oppitz U, Maessen D, Zunterer H, Richter S, Flentje M. 3D-recurrencepatterns of glioblastomas after CT-planned postoperative irradiation. Radiother Oncol. 1999:53(1):53-7.

\section{Submit your next manuscript to BioMed Central and we will help you at every step:}

- We accept pre-submission inquiries

- Our selector tool helps you to find the most relevant journal

- We provide round the clock customer support

- Convenient online submission

- Thorough peer review

- Inclusion in PubMed and all major indexing services

- Maximum visibility for your research

Submit your manuscript at www.biomedcentral.com/submit 